OKHEP-01-08

\title{
Bulk versus brane running couplings
}

\author{
Kimball A. Milton \\ Department of Physics and Astronomy, \\ The University of Oklahoma, Norman 73019 USA \\ Sergei D. Odintsovi] \\ Instituto de Fisica de la Universidad de Guanajuato, \\ Lomas del Bosque, Leon, MEXICO \\ Sergio Zerbinif \\ Department of Physics and Gruppo Collegato INFN \\ University of Trento, ITALY
}

(Dated: October 26, 2018)

\begin{abstract}
A simplified higher dimensional Randall-Sundrum-like model in 6 dimensions is considered. It has been observed previously by Goldberger and Wise that in such a self-interacting scalar theory on the bulk with a conical singularity there is mixing of renormalization of $4 \mathrm{~d}$ brane couplings with that of the bulk couplings. We study the influence of the running bulk couplings on the running of the $4 \mathrm{~d}$ brane couplings. We find that bulk quantum effects may completely alter the running of brane couplings. In particular, the structure of the Landau pole may be drastically altered and non-asymptotically free running may turn into asymptotically safe (or free) behavior.
\end{abstract}

PACS numbers: 11.10.Hi, 04.50.+h, 11.10.Kk

\footnotetext{
*milton@mail.nhn.ou.edu

todintsov@ifug5.ugto.mx; Tomsk State Pedagogical University, Tomsk, RUSSIA

zerbini@science.unitn.it
} 


\section{INTRODUCTION}

Recently Randall-Sundrum brane-world models [1] have attracted a great deal of interest in particle physics and phenomenology. One reason is that such models may resolve the hierarchy problem in a quite natural way. Moreover, they may lead to some interesting changes in the phenomenology of the Standard Model and related theories.

A typical modern approach to quantum high energy theory is to consider quantum fields on a higher dimensional manifold (the bulk) in the presence of extended defects (the boundaries). For example, we could consider a 3-brane localized in a 4-dimensional submanifold. On the bulk manifold, as well as on the brane, there exist divergences which result in the running of coupling constants in the standard way. However, it has been known for some time that for spacetimes with boundaries there are not only the usual volume coupling constants but also there are surface coupling constants [2]. Of course, these influence each other; for example, volume interactions are reflected in surface terms, etc [3]. However, so far no drastic consequences have been discovered. Nevertheless, it could be that the situation is different in some brane-world models. For example, one may wonder how the running of the bulk couplings influences the running on the brane and vice versa. With regard to this issue, a very simple $6 \mathrm{~d}$ model with a $4 \mathrm{~d}$ brane has been proposed in Ref. 四. However, only renormalization of bulk and brane coupling constants has been investigated there. Here we reconsider this model, making use of heat-kernel techniques and perform estimates of the running of bulk and brane couplings. It will be seen that the bulk running couplings can significantly influence the running of the $4 \mathrm{~d}$ cosmological constant, $4 \mathrm{~d}$ mass and $4 \mathrm{~d}$ self-scalar interaction. In particular, instead of a constant behavior of the cosmological constant (in the sense of the renormalization group), we find a running, such that the cosmological constant increases with energy. The Landau pole for the brane mass and the scalar self-interaction is drastically altered by the bulk running couplings, and in general becomes a cut.

\section{THE MODEL}

Our toy model consists of a massive Euclidean self-interacting scalar in a 6-dimensional space with a conical singularity, due to the presence of 3-brane. The metric is chosen to be

$$
d s_{6}^{2}=d r^{2}+r^{2} d \theta^{2}+d s_{0}^{2}
$$

where $d s_{0}^{2}$ is the 4-dimensional flat metric, the brane is located at $r=0$, and $\theta$ has a period $\beta, \beta$ being the deficit angle of the cone. When $\beta=2 \pi / N, N$ a positive integer, one is dealing with a less singular manifold, namely an orbifold, while for $N=1, \beta=2 \pi$, one has the smooth 2-dimensional plane. The action reads

$$
S=\int d^{6} x \sqrt{g}\left[\phi \frac{1}{2}\left(-\square_{6}+m^{2}\right) \phi+V(\phi)\right]+\int d^{4} x W(\phi),
$$

where $V(\phi)=\frac{g_{4}}{4 !} \phi^{4}+\ldots$ denotes a series of scalar bulk couplings. We also introduce a "surface" term which depends on surface scalar couplings

$$
W(\phi)=\left[\lambda_{0}+\frac{\lambda_{2}}{2} \phi^{2}+\frac{\lambda_{4}}{4 !} \phi^{4}+\ldots\right]
$$


namely it may contain a brane tension $\lambda_{0}$, a brane mass $\lambda_{2}$, a $\phi^{4}$ coupling $\lambda_{4}$, as well as higher terms. As we will see, these surface terms are necessary because we are dealing with a manifold with a conical singularity (see also Ref. [5]). We also assume that the brane is not dynamical, namely we are dealing with a rigid brane, and therefore we neglect the brane kinetic term.

The total one-loop action operator consists of a bulk part and a contact delta-function singular potential. First, we discuss briefly the problems associated with this latter contact term. For a nice discussion from a diagrammatic point of view, see the approach contained in Ref. [4]. Here one may follow the standard and well-known argument used in nonrelativistic quantum mechanics and put on a rigorous basis in Refs. [6, 7] (see also references cited therein).

Briefly, the issue to be addressed is based on the following remarks. When one has to evaluate the Green's functions (i.e. two-point functions or correlation functions) related to a Laplace operator "perturbed" by a delta-function potential, typically one is forced to deal with the product of a delta-function distribution and a two point-function, which can have singularities on the support of the delta function. Only in codimension 1 (RS-like models) is this singularity absent, since then the two-point function is not singular at the coincidence limit of the two points.

The method to be used [6] consists in the regularization of the delta function by means of a sequence of smooth functions depending on a cutoff parameter. The key point is to assume that the bare coupling constants (which can be thought of as strengths of the terms in the delta-function potential) also depend on the regularization parameter. Then, one can easily solve the functional equation for the two-point function within this regularization. In order to remove the cutoff parameter dependence, one has to assume that the contact divergence, present if the codimension is bigger than one, is cured by a suitable compensating behavior of the coupling. If the codimension is two, the divergence is only logarithmic and, for dimensional reasons, there is room for the appearance of an arbitrary renormalization parameter $\mu$. In general, this happens when the codimension is even. As a result, the renormalized coupling constants will depend on $\mu$ and it is not difficult to demonstrate that they must satisfy renormalization group equations.

\section{ONE-LOOP CORRECTION AND RENORMALIZATION GROUP}

The one-loop correction is determined by the total one-loop fluctuation operator, which reads

$$
L_{6}=-\square_{\beta}-\square_{4}+M^{2}+W^{\prime \prime}(\Phi) \delta^{(2)}(x),
$$

where $\square_{\beta}$ is the 2-dimensional Laplacian on the cone, $\Phi$ is the background field and $M^{2}=$ $m^{2}+V^{\prime \prime}(\Phi)$ is an effective mass.

We shall make use of zeta-function regularization and related heat-kernel techniques (see, for example Refs. [8, 9]). Within the one-loop approximation, we have to evaluate the zetafunction at zero, namely $\zeta\left(0 \mid L_{6}\right)$, since this quantity gives rise to the one-loop divergences and governs the one-loop beta functions. There are also contributions due to the conical singularity and the brane delta-function contribution, which gives additive contributions to $\zeta\left(0 \mid L_{6}\right)$, which have been diagrammatically evaluated in Ref. [4]. 
The zeta-function for the operator $L_{6}$ is defined by

$$
\zeta\left(s \mid L_{6}\right)=\frac{1}{\Gamma(s)} \int_{0}^{\infty} d t t^{s-1} \operatorname{Tr} e^{-t L_{6}} .
$$

In the presence of the conical singularity and delta-function potential, one has for small $t$

$$
\begin{aligned}
\operatorname{Tr} e^{-t L_{6}}=\int & d^{6} x \frac{e^{-t M^{2}}}{(4 \pi)^{3} t^{3}}\left[1+b_{2}(x) t^{2}+b_{3}(x) t^{3}+O\left(t^{4}\right)\right] \\
& +\int d^{4} x \frac{e^{-t M^{2}}}{(4 \pi)^{2} t^{2}}\left(I_{\beta}+I_{W}\right)\left[1+b_{2}(x) t^{2}+b_{3}(x) t^{3}+O\left(t^{4}\right)\right]
\end{aligned}
$$

where $b_{2}(x)$ and $b_{3}(x)$ depend only on the derivative of the effective mass and are given by

$$
\begin{aligned}
& b_{2}(x)=-\frac{1}{6} \square M^{2}, \\
& b_{3}(x)=-\frac{1}{60} \square^{2} M^{2}+\frac{1}{12} \partial_{\mu} M^{2} \partial^{\mu} M^{2} .
\end{aligned}
$$

We also have included the boundary terms due to the conical singularity and the deltafunction contact term. The first depends on the numerical quantity 10

$$
I_{\beta}=\frac{1}{12}\left(\frac{2 \pi}{\beta}-\frac{\beta}{2 \pi}\right)
$$

Of course, this boundary term is absent if $\beta=2 \pi, I_{\beta}=0$, namely in the absence of the conical singularity.

The contact contribution depends on the quantity $W^{\prime \prime}$, and in the perturbative regime, corresponding to $W^{\prime \prime}$ "small," is, as a function of the background field, simply given by

$$
W^{\prime \prime}(\Phi)=\left[\lambda_{2} \Phi^{2}+\frac{1}{2} \lambda_{4} \Phi^{4}+\frac{1}{4 !} \lambda_{6} \Phi^{6}+\ldots\right],
$$

The analytic continuation of the zeta function to $s=0$ is

$$
\begin{aligned}
\zeta\left(0 \mid L_{6}\right)=\int & d^{6} x \frac{1}{(4 \pi)^{3}}\left[-\frac{\left(M^{2}\right)^{3}}{6}-b_{2}(x) M^{2}+b_{3}(x)\right] \\
& +\int d^{4} x \frac{1}{(4 \pi)^{2}}\left(I_{\beta}+I_{W}\right)\left[\frac{\left(M^{2}\right)^{2}}{2}+b_{2}(x)\right] .
\end{aligned}
$$

The heat kernel coefficients $a_{n}$ are defined by the small- $t$ expansion of the heat kernel, $(d$ is the dimension of the manifold, here $d=6$ )

$$
\operatorname{Tr} e^{-t L_{6}} \sim \frac{1}{(4 \pi t)^{d / 2}} \sum_{n} a_{n} t^{n}, \quad t \rightarrow 0+,
$$

so the integrand in the first term of the above expression is the $a_{3}(x)$ coefficient, which reads

$$
a_{3, \text { bulk }}(x)=-\frac{\left(M^{2}\right)^{3}}{6}+\frac{M^{2} \square M^{2}}{6}+\frac{\partial_{\mu} M^{2} \partial^{\mu} M^{2}}{12}-\frac{\square^{2} M^{2}}{60} .
$$


For the sake of completeness, we note that the $a_{2}(x)$ coefficient, which is relevant in four dimensions, is

$$
a_{2}(x)=\frac{\left(M^{2}\right)^{2}}{2}-\frac{\square M^{2}}{6}
$$

These expressions actually define the structure of the one-loop bulk and surface divergences. It is already clear that the theory under consideration is not renormalizable at the one-loop level! In such a situation, we will be interested in obtaining the one-loop renormalization for "renormalizable" bulk interactions which will appear also in the renormalization of brane couplings. These are the mass and four-scalar bulk couplings. They are actually defined by the term in $a_{3, \text { bulk }}$ cubic in the effective mass. The corresponding one-loop renormalization group ( $\mathrm{RG}$ ) equations have the form

$$
\begin{aligned}
\frac{d m^{2}}{d t} & =c_{1} m^{4} g_{4} \\
\frac{d g_{4}}{d t} & =c_{2} m^{2} g_{4}^{2}
\end{aligned}
$$

where

$$
c_{1}=\frac{1}{2} \frac{1}{(4 \pi)^{3}}, c_{2}=\frac{3}{(4 \pi)^{3}}
$$

and $t$ stands for $\ln \frac{\mu}{\mu_{0}}$. The solutions of these equations are not difficult to find, since their product $p(t)=g_{4}(t) m^{2}(t)$ satisfies $\left(c=c_{1}+c_{2}\right)$

$$
\frac{d p}{d t}=c p^{2}
$$

As a result, $\left(p_{0}=p(0)\right)$

$$
\begin{aligned}
p(t) & =\frac{p_{0}}{1-c p_{0} t}, \\
m^{2}(t) & =m^{2}(0)\left(1-c p_{0} t\right)^{-c_{1} / c}, \\
g_{4}(t) & =g_{4}(0)\left(1-c p_{0} t\right)^{-c_{2} / c} .
\end{aligned}
$$

As $p_{0}$ is positive, we have to consider the regime $1>c p_{0} t$. When the denominator in above equations becomes zero we see the appearance of a Landau pole in $p(t)$ in terms of the initial mass and self-interaction. (In $m^{2}$ and $g_{4}$ the singularity is a branch point.) As one sees, the infrared (IR) $(t \rightarrow-\infty)$ behavior of above RG couplings is asymptotically free (AF).

A more interesting scenario emerges if we recognize that our observable physics is defined on a $4 \mathrm{~d}$ brane. Whatever the physics is in six dimensions, we require that it be sensible for a 4d-brane observer. For example, let us imagine that we could take the wrong sign for the scalar self-interaction in six dimensions. Then, the RG equation for $g_{4}$ appears with the opposite sign. In other words, the RG solution for $g_{4}$ appears with a positive sign in the denominator and becomes asymptotically free in the UV. The same occurs with the RG mass. Thus, we may search for consequences on the brane of both AF and non-AF RG solutions. 
Proceeding onwards, we see that the regularized one-loop effective action reads

$$
\ln \operatorname{det} \frac{L_{6}}{\mu^{2}}(\varepsilon)=-\frac{\mu^{2 \varepsilon}}{2} \int_{0}^{\infty} d t t^{\varepsilon-1} \operatorname{Tr} e^{-t L_{6}}=-\frac{\mu^{2 \varepsilon}}{2} \Gamma(\varepsilon) \zeta\left(\varepsilon \mid L_{6}\right),
$$

where an arbitrary mass scale $\mu$ has been introduced to keep each expression dimensionless. Thus, for small $\varepsilon$,

$$
\ln \operatorname{det} \frac{L_{6}}{\mu^{2}}(\varepsilon)=-\frac{1}{2}\left[\frac{\zeta\left(0 \mid L_{6}\right)}{\varepsilon}+\left(\ln \mu^{2}+\gamma\right) \zeta\left(0 \mid L_{6}\right)+\zeta^{\prime}\left(0 \mid L_{6}\right)+\mathcal{O}(\varepsilon)\right] .
$$

One can see that due to the presence of the conical singularity, there are "brane" surface and contact contributions in the divergence as well as in the finite part depending on the scale $\mu^{2}$. These surface contributions have to be added to the bulk counterterm in order to remove the additional ultraviolet divergences related to the conical singularity and contact term. These additional terms also modify the one-loop renormalization group equations and depend on the bulk potential $V(\phi)$ and the brane potential $W(\phi)$.

Let us now write the RG equations for the surface (brane) couplings which were derived in Ref. 四. For the special choice of the deficit angle $\beta=\pi$ (a $Z_{2}$ orbifold), they read at one-loop,

$$
\begin{aligned}
\frac{d \lambda_{0}}{d t} & =\frac{m^{4}}{256 \pi^{2}}-\frac{m^{4} \lambda_{2}}{64 \pi^{3}}, \\
\frac{d \lambda_{2}}{d t} & =\frac{\lambda_{2}^{2}}{\pi}+\frac{m^{2} g_{4}}{128 \pi^{2}}-\frac{m^{4} \lambda_{4}}{64 \pi^{3}} .
\end{aligned}
$$

We also include the renormalization group equation for the coupling constant related to the brane four-point function, the last term of which was not given explicitly in Ref. [⿴囗木:

$$
\frac{d \lambda_{4}}{d t}=\frac{4 \lambda_{2} \lambda_{4}}{\pi}+\frac{3 g_{4}^{2}}{128 \pi^{2}}-\frac{m^{4} \lambda_{6}}{64 \pi^{3}} .
$$

In the above equations the last terms have their origin in the delta-function contribution (9).

First, let us find the solutions of these RG equations when the one-loop corrections are not included, i.e., we drop the bulk terms involving $m$ and $g_{4}$. Note that $\lambda_{0}$ is then a constant. The other two couplings are:

$$
\begin{aligned}
& \lambda_{2}(t)=\frac{\lambda_{2}(0)}{1-\frac{\lambda_{2}(0)}{\pi} t} . \\
& \lambda_{4}(t)=\lambda_{4}(0)\left(1-\frac{\lambda_{2}(0) t}{\pi}\right)^{-4} .
\end{aligned}
$$

As one can see, we find non-asymptotically free behavior for these coupling constants. At high energies there is a Landau pole defined by the initial RG mass. At the same time, as usual, in the IR limit we have AF behavior for both coupling constants.

In order to investigate the role of the one-loop quantum corrections, let us consider only the surface terms and neglect the contact terms. That is, we consider the truncated equations 
(with $\left.p=m^{2} g_{4}\right)$

$$
\begin{aligned}
\frac{d \lambda_{0}}{d t} & =\frac{m^{4}}{256 \pi^{2}}, \\
\frac{d \lambda_{2}}{d t}-\frac{\lambda_{2}^{2}}{\pi} & =\frac{p}{128 \pi^{2}}, \\
\frac{d \lambda_{4}}{d t}-\frac{4 \lambda_{2} \lambda_{4}}{\pi} & =\frac{3 g_{4}^{2}}{128 \pi^{2}} .
\end{aligned}
$$

The first of these equations can be easily integrated,

$$
\lambda_{0}(t)-\lambda_{0}(0)=\frac{m^{4}(0)}{256 \pi^{2}} \frac{1}{\left(c_{2}-c_{1}\right) p_{0}}\left[1-\left(1-c p_{0} t\right)^{\frac{c_{2}-c_{1}}{c}}\right] .
$$

This reveals a very interesting running behavior for the effective cosmological constant. In the absence of the bulk contribution the cosmological constant was unchanged from its initial RG value. However, after taking into account the bulk running, the evolution of the cosmological constant is completely altered. As long as $\left(c_{2}-c_{1}\right) / c$ is not an integer, which is the case here, this solution still possesses a branch point at a suitably large value of $t$; moreover, it is not asymptotically free in either the IR nor in the UV, but for small $t$ grows linearly with $t$. Because $c_{2}>c_{1}$, it becomes large in both the IR and UV limits.

The third equation can be integrated once a solution of the second is known. Although the latter is a nonlinear inhomogeneous differential equation belonging to the Riccati class, the general solution of which is not known, in our specific case, one can find an explicit and exact solution (see Appendix).

Let us first consider two limits. For small $t$ we can develop a perturbative (power series) solution in $t$,

$$
\lambda_{2}(t)=\lambda_{2}(0)+\sum_{n=1}^{\infty} d_{n} t^{n}
$$

where from Eqs. (22b) and (17a) we easily find

$$
\begin{aligned}
& d_{1}=b+\frac{\lambda_{2}(0)^{2}}{\pi} \\
& d_{2}=\frac{\alpha b^{2}}{2 \pi}+\frac{b}{\pi} \lambda_{2}(0)+\frac{1}{\pi^{2}} \lambda_{2}(0)^{3} \\
& d_{3}=\frac{\alpha^{2} b^{3}}{3 \pi^{2}}+\frac{b^{2}}{3 \pi}+\frac{\alpha b^{2}}{3 \pi^{2}} \lambda_{2}(0)+\frac{4 b}{3 \pi^{2}} \lambda_{2}(0)^{2}+\frac{\lambda_{2}(0)^{4}}{\pi^{3}}
\end{aligned}
$$

and so on. Here we have defined

$$
b=\frac{p_{0}}{128 \pi^{2}}, \quad \alpha=\frac{\pi}{b} p_{0} c
$$

where in our case $\alpha=7$.

The other interesting limit is large $t$, that is $t \rightarrow-\infty$ if $p_{0}>0$ or $t \rightarrow \infty$ if $p_{0}<0$. Then because

$$
p(t) \sim-\frac{1}{c t}, \quad p_{0} t \rightarrow-\infty
$$


we find by achieving the only balance possible in Eq. (22b) that

$$
\lambda_{2}(t) \sim \pm \sqrt{\frac{|b| \pi}{1+|b| \alpha t / \pi}}, \quad p_{0}<0, t \rightarrow \infty .
$$

As one sees the Landau pole found in Eq. (21a) seems to disappear from our running behavior. The mass is decreasing with the RG scale.

Finally, the solution of Eq. (22d) reads

$$
\begin{gathered}
\lambda_{4}(t)=\frac{3}{128 \pi^{2}} e^{\frac{4}{\pi} \int_{0}^{t} d t^{\prime} \lambda_{2}\left(t^{\prime}\right)}\left[\int_{0}^{t} d t^{\prime} e^{-\frac{4}{\pi} \int_{0}^{t^{\prime}} d t_{1} \lambda_{2}\left(t_{1}\right)} g_{4}^{2}\left(t^{\prime}\right)\right] \\
+\lambda_{4}(0) e^{\frac{4}{\pi} \int_{0}^{t} d t^{\prime} \lambda_{2}\left(t^{\prime}\right)}
\end{gathered}
$$

We can easily insert the perturbative solution (24) into this expression to obtain the small $t$ behavior of $\lambda_{4}$. The leading term is

$$
\lambda_{4}(t)=\lambda_{4}(0)+\left[\frac{3}{128 \pi^{2}} g_{4}(0)+\frac{4}{\pi} \lambda_{2}(0) \lambda_{4}(0)\right] t+\mathcal{O}\left(t^{2}\right) .
$$

This, of course, immediately follows from Eq. (220).

More interesting again is the large $t$ behavior. The leading behavior can be readily determined from Eq. (22) by seeing, when the large $t$ behavior coming from Eq. (17d) is inserted, which two terms in the differential equation can balance. It is clear that the only balance possible is between the second and third terms, so we immediately find

$$
\lambda_{4}(t) \sim \frac{3}{32 \sqrt{2 \pi\left|p_{0}\right|}} g_{4}(0)^{2}\left(1-c p_{0} t\right)^{\frac{1}{2}-\frac{2 c_{2}}{c}}, \quad p_{0}<0, t \rightarrow \infty .
$$

Since $\frac{1}{2}-\frac{2 c_{2}}{c}=-\frac{5}{14}<0$, this says that in the UV the four-scalar coupling constant decreases. This is in the contrast with the non-AF behavior seen in Eq. (21b) without taking into account the bulk running!

These results are generalized by using the results of the analysis given in Appendix. According to the general solution it follows that if we started from non-AF bulk running coupling (the case $p_{0}>0$ ) then the structure of the Landau singularity for the brane mass (and here also for the brane four-coupling) is completely changed! Previously in Eq. (21a), it was defined by the initial RG value of the brane mass $\lambda_{2}(0)$. Now, there are an infinite number of singularities defined by the initial RG values of the bulk mass and the bulk fourscalar coupling! That is, there is a singularity (in general, a branch point) at $t=t_{0}$, where

$$
t_{0}=\frac{1}{c p_{0}}=\frac{\pi}{\alpha b}
$$

and poles at $t=t_{n}$,

$$
t_{n}=t_{0}-\frac{\alpha}{4} z_{n}^{2}
$$

where $z_{n}$ is the $n$th zero of a Bessel function. Consequently, if one starts from the AF behavior for the bulk couplings (case $p_{0}<0$ ), there are no singularites for $t>0$. That 
is, the Landau pole of the brane couplings has been eaten by bulk quantum effects. The running behavior of the brane couplings becomes completely asymptotically safe! Of course one could present corresponding numerical results for all couplings. We will not do this as the model under discussion is over-simplified and hardly realistic.

The important lesson which follows from our discussion is follows. Even though the brane observer does not know much about the bulk wherein his brane is embedded, there exists a clear influence of bulk quantum physics on the quantum physics on the brane. In particular, one may conjecture that there may be the realistic (Standard Model-like) theories where observable running effects like asymptotic freedom are provided by bulk running couplings. It is now a challenge to construct such brane-world models.

The research of KAM has been supported in part by a grant from the US Department of Energy (\#DE-FG03-98ER41066), that of SDO in part by CONACyT (CP, Ref. 990356) and in part by INFN, and that of SZ by INFN. SZ thanks G. Cognola for helpful discussions.

\section{SOLUTION OF RICCATI EQUATION}

In this Appendix, we show how the Riccati equation (22b) can be solved. According to the general theory of ordinary differential equations (see, for example, Ref. [11]), it is known

that solutions of the Riccati equation can be found as soon as one is able to find solutions of a related linear second order differential equation. In our case, we may rewrite Eq. (22b) in the form

$$
\lambda_{2}^{\prime}(x)+\frac{\lambda_{2}^{2}(x)}{\pi c p_{0}}+\frac{1}{128 \pi^{2} c x}=0 .
$$

with $^{\prime}=\frac{d}{d x}$ and $x=1-c p_{0} t$. The associated linear second order equation simply reads

$$
\frac{d^{2} u}{d^{2} x}+\frac{B u}{x}=0,
$$

with $B=\frac{\pi}{\alpha^{2} b}$. The corresponding solution of the Riccati equation is

$$
\lambda_{2}(x)=\alpha b \frac{d}{d x} \ln u(x) .
$$

Fortunately, Eq. (34) can be reduced to a Bessel equation. In fact, putting $z=2 \sqrt{B x}$ and

$$
u(x(z))=\frac{z}{2 \sqrt{B}} Z_{1}(z),
$$

a simple computation leads to

$$
\frac{d^{2} Z_{1}}{d^{2} z}+\frac{1}{z} \frac{d Z_{1}}{d z}+\left(1-\frac{1}{z^{2}}\right) Z_{1}=0
$$

which is the Bessel equation of order $\nu=1$.

The solution to Eq. (37) is

$$
Z_{1}(z)=J_{1}(z)+A N_{1}(z)
$$


where $J_{1}$ is the Bessel function of the first kind of order 1 , and $N_{1}$ is the Neumann function of order 1. The arbitrary constant of integration is $A . \lambda_{2}$ is now given by Eq. (35) with

$$
u(x)=\sqrt{x} Z_{1}(2 \sqrt{B x}) .
$$

The constant $A$ is determined by the initial value of $\lambda_{2}$ :

$$
\lambda_{2}(t=0)=\left.\alpha b \frac{d}{d x} \ln u(x)\right|_{x=1} .
$$

What are the singularities of $\lambda$ ? If $A=0, Z_{1}$ has a zero at $z=0$; otherwise it has a branch point there. This means that there is a singularity at $x=0$ or at

$$
t_{0}=\frac{1}{c p_{0}}
$$

This is the reflection of the Landau pole in $p(t)$, Eq. (17a). But there an an infinite number of other singularites of $\lambda_{2}$, occurring where $Z_{1}$ possesses zeros; call these zeroes $z_{n}$. (Asymptotically,

$$
z_{n}=\arctan \frac{1+A}{1-A}+n \pi
$$

where the arctangent is the principal value and $n$ is an integer.) The corresponding values of $t_{n}$ are

$$
t_{n}=t_{0}-\frac{\alpha}{4} z_{n}^{2}
$$

Thus, if $p_{0}>0$, to the right of $t=0$ there are a finite number of singularities, but an infinite number to the left; for $p_{0}<0$ all the singularities occur for $t<0$. So let us examine the latter situation. If $p_{0}<0, B<0$, so we write the solution in terms of modified Bessel functions (Bessel functions of imaginary argument),

$$
\begin{aligned}
Z_{1}(i z) & =I_{1}(z)+A K_{1}(z), \\
u(x) & =\sqrt{x}\left[I_{1}(2 \sqrt{|B| x})+A K_{1}(2 \sqrt{|B| x})\right] .
\end{aligned}
$$

Up to a constant factor, asymptotically,

$$
u(x) \sim e^{2 \sqrt{|B| x}}+A e^{-2 \sqrt{|B| x}}, \quad x \rightarrow \infty .
$$

Thus we find the leading behavior

$$
\lambda_{2}(t) \sim-\sqrt{\frac{\pi|b|}{1-c p_{0} t}}, \quad p_{0}<0, t \rightarrow \infty .
$$

This is consistent with the asymptotic result (28), and therefore also implies the behavior seen in Eq. (31).

[1] L. Randall and R. Sundrum, Phys. Rev. Lett. 83 (1999) 3370; 83 (1999) 4690. 
[2] S.D. Odintsov, Class. Quant. Grav. 7 (1990) 445; S.D. Odintsov and A. Wipf, Phys. Lett. B356 (1995) 26.

[3] G. Tsoupros, Class. Quant. Grav. 17 (2000) 2255.

[4] W. D. Goldberger and M. B. Wise, Renormalization Group Flows for Brane Couplings CALT68-2327, hep-th/0104170.

[5] H. Georgi, A.K. Grant and G. Hailu, hep-ph/0012379.

[6] F.A. Berezin and L.D. Faddeev, Soviet Math. Dokl. 2 (1961) 372.

[7] S. Albeverio, F. Gesztesy, R. Hoegh-Krohn, H, Holden, Solvable Models in Quantum Mechanics. Springer-Verlag (1988).

[8] E. Elizalde, S.D. Odintsov, A. Romeo, A.A. Bytsenko and S. Zerbini, Zeta regularization techniques with applications, World Scientific, 1994.

[9] A.A. Bytsenko, G. Cognola, L. Vanzo and S. Zerbini, Phys. Reps. 269, (1996) 1.

[10] J. Dowker, J. Phys. A10, (1977) 115; G.Cognola, K. Kirsten and L.Vanzo, Phys. Rev. D 49 (1994) 1029; D.V. Fursaev, Class. Quantum Grav. 11 (1994)11; S. Zerbini, G. Cognola and L.Vanzo, Phys. Rev. D 54 (1996) 2699.

[11] E.L. Ince, Ordinary Differential Equations, Dover, New York, 1944. 